Pacific

Journal of

Mathematics

\title{
THE FUNCTIONAL EQUATION \\ AND BEYOND ENDOSCOPY
}

P. EDWARD HERMAN 


\title{
THE FUNCTIONAL EQUATION AND BEYOND ENDOSCOPY
}

\author{
P. EDWARD HERMAN
}

Dedicated to the memory of Jonathan Rogawski

\begin{abstract}
In his paper "Beyond endoscopy," Langlands tries to understand functoriality via poles of $L$-functions. This paper further investigates the analytic continuation of an $L$-function associated to a $G L_{2}$ automorphic form through the trace formula. Though the usual way to obtain the analytic continuation of an $L$-function is through its functional equation, this paper shows that by simply assuming the trace formula, the functional equation of the $L$-function may be recovered. This paper is a step towards understanding the analytic continuation of the $L$-function at the same time as capturing information about functoriality.

From the perspective of analytic number theory, obtaining the functional equation from the trace formula implies that Voronoi summation should in general be also a consequence of the trace formula.
\end{abstract}

\section{Beyond endoscopy}

Let $\mathbb{A}_{\mathbb{Q}}$ be the ring of adeles of $\mathbb{Q}$, and $\pi$ be an automorphic cuspidal representation of $\mathrm{GL}_{2}\left(\mathbb{A}_{\mathbb{Q}}\right)$. We define $m(\pi, \rho)$ to be the order of the pole at $s=1$ of $L(s, \pi, \rho)$, where $\rho$ is a representation of the dual group $\mathrm{GL}_{2}(\mathbb{C})$.

Langlands proposes the study of

$$
\lim _{X \rightarrow \infty} \sum_{\pi} \frac{1}{X} \operatorname{tr}(\pi)(f) \sum_{p \leq X} \log (p) a(p, \pi, \rho) .
$$

Here $f$ is a nice test function on $\mathrm{GL}_{2}\left(\mathbb{A}_{\mathbb{Q}}\right), \operatorname{tr}(\pi)(f)$ is the trace of the operator defined by $f$ on $\pi$, and $a(p, \pi, \rho)$ is the $p$-th Dirichlet coefficient of $L(s, \pi, \rho)$. The quantity

$$
\lim _{X \rightarrow \infty} \frac{1}{X} \sum_{p \leq X} \log (p) a(p, \pi, \rho)
$$

is equal to $m(\pi, \rho)$.

MSC2010: 11F12, 11F66, 11F72, 11L05.

Keywords: trace formula, $L$-functions, functional equation, automorphic forms. 
Therefore, summing over the range of representations $\pi$ will project only on to the ones which have nontrivial multiplicity. The tool used to study this sum over the spectrum of forms $\pi$ is the trace formula. Ultimately, one gets from use of the trace formula a sum over primes and conjugacy classes, and hopes by analytic number theory techniques to take the limit. One hopes that after getting the limit, one can decipher and construct the $L$-functions having nontrivial multiplicity of the pole at $s=1$. Sarnak [2001] addresses (1-1) for $\rho=s t d$, the standard representation. He points out that such a computation can be done, but the tools used for the study of sums of primes is limited, and this problem is perhaps more tractable if rather studied over the sum of integers.

Sarnak's idea then is to evaluate

$$
\lim _{X \rightarrow \infty} \sum_{\pi} \frac{1}{X} \operatorname{tr}(\pi)(f) \sum_{n \leq X} a(n, \pi, \rho) .
$$

This should "detect," rather than the multiplicities of the poles, the residue of the poles of the associated $L$-functions. As well, instead of using the Arthur-Selberg trace formula, he uses the Petersson-Kuznetsov trace formula, which is a special case of the relative trace formula [Knightly and Li 2006a]. One advantage of the relative trace formula is that the spectrum contains only generic representations, so we avoid the task of excising the trivial representation as in [Frenkel et al. 2010]. As well, the geometric side of the relative trace formula has a nice "streamlined" appearance as a sum of Kloosterman sums. This is in comparison to the ArthurSelberg trace formula, which has orbital integrals associated to different conjugacy classes for which the analysis of each class could be different.

The disadvantage to the relative trace formula is that each automorphic representation $\pi$ on the spectral side of the trace formula is weighted by a factor $L(1, \pi, a d)^{-1}$, which is the adjoint representation of $\pi$ evaluated at $s=1$. This can perhaps make matching two different trace formulas more difficult. Another disadvantage of using the relative trace formula is that the Arthur-Selberg trace formula is in much better shape to generalize to other groups. Namely, one now has full use of the stable trace formula due to the proof of the "fundamental lemma" by Ngô [2010]. With the stable trace formula, one can compare stable conjugacy classes for different groups (specifically endoscopic groups), from which one can then compare automorphic representations for the respective groups.

However in our case of studying $\mathrm{GL}_{2}$, the disadvantages seem minimal, and in fact the crucial exponential sums one encounters in either trace formula are the same. Sarnak [2001] made some points on the essential differences of the geometric sides of the two trace formulas. Also, in the case of $\mathrm{GL}_{2}$, the stable trace formula is the same as the Arthur-Selberg trace formula, so one should not expect an advantage of one trace formula over another. 
1.1. Sarnak's analysis for $\rho=s t d$. The obvious first example to test Langlands's beyond endoscopy idea on is for the standard representation. In this case we do not expect the $L$-functions to have any poles except for the continuous spectrum, but in this case there are not any poles as the spectrum is not spectrally isolated. So we expect in the case of $\rho=s t d$ that

$$
\lim _{X \rightarrow \infty} \sum_{\pi} \frac{1}{X} \operatorname{tr}(\pi)(f) \sum_{n \leq X} a(n, \pi, s t d)=0 .
$$

Sarnak uses the classic Petersson-Kuznetsov trace formula instead of using the adelic language. To go from (1-3) to a classic approach, one can follow the great expository article of Rogawski [1994] or the book of Knightly and Li [2006b]. Then for an automorphic form $f$ with normalized Fourier coefficients $a_{n}(f)$ associated to a representation $\pi$, Sarnak [2001] showed, up to some weight factors needed in the trace formula, that

$$
\sum_{n \leq X} \sum_{f} a_{n}(f) g(n / X)=O\left(X^{-A}\right)
$$

for any $A>0$. Here $X$ is a large fixed parameter and $g \in C_{0}^{\infty}\left(\mathbb{R}^{+}\right)$is used for "smoothing" the $n$-sum. Why is this smoothing needed? It is certainly not essential, but when one goes to the geometric side of the trace formula to get the bound (1-4), one requires freedom to apply analytic manipulations (interchanging sums, Fourier transforms, and so on). With the smoothing function $g$, these problems are removed and one can focus on the central issue of the arithmetic, which is the true difficulty in these problems. One can recover the left hand side of (1-3) by applying techniques in [Iwaniec 1984]. For completeness, we will reproduce Sarnak's argument in the appendix.

1.2. Results of the paper. Clearly (1-4) is a stronger result than (1-3), and up to using Hecke operators, is equivalent to $L(s, f)=\sum_{n=1}^{\infty} a_{n}(f) / n^{s}$ having analytic continuation to the complex plane. We see the analytic continuation of the left hand side of (1-4) by Mellin inversion. By applying Mellin inversion to (1-4) we get

$$
\frac{1}{X} \sum_{f} \sum_{n \leq X} g(n / X) a_{n}(f)=\frac{1}{2 \pi i} \int_{\sigma-i \infty}^{\sigma+i \infty} G(s)\left[\sum_{f} L(s, f)\right] X^{s} d s,
$$

where $G(s)=\int_{0}^{\infty} g(x) x^{s-1} d x$ is the Mellin transform with $\sigma>2$ to ensure the convergence of the integral. Using the right hand side of (1-4) we know that the contour in (1-5) can be shifted (using decay properties of $G(s)$ ) to $\sigma=-A, A>0$. So in Sarnak's application of the trace formula to get (1-4) we indirectly applied a functional equation of the $L$-function for each automorphic form $f$ in our spectral 
sum. Can we actually see directly the functional equation via manipulations on the geometric side of the trace formula? In other words, can we show directly via the trace formula that

$$
\begin{aligned}
\frac{1}{2 \pi i} \int_{\sigma-i \infty}^{\sigma+i \infty} G(s)\left[\sum_{f} L(s, f)\right] X^{s} d s & \\
& =\frac{1}{2 \pi i} \int_{\sigma-i \infty}^{\sigma+i \infty} G(s)\left[\sum_{f} \frac{i^{k} \gamma(f, 1-s) L(f, 1-s)}{\gamma(f, s)}\right] X^{s} d s ?
\end{aligned}
$$

We will prove this equality and get the functional equation for a fixed automorphic form $f$ in this note.

There are two other methods we mention that also get the analytic continuation of an automorphic form on $\mathrm{GL}_{n}$; both use integral representations. The first method is associated to Jacquet and Langlands [1970] (who followed Hecke [1918; 1920]), and expresses the standard $L$-function as an adelic integral of an explicitly chosen vector in the space of the associated automorphic representation. The second method is of a certain integral representation constructed by Godement and Jacquet [1972], which is inspired by Tate's construction [1967] for GL $_{1}$.

One can consider these two methods as easier ways to get the functional equation for a $\mathrm{GL}_{2}$ automorphic form, but in consideration of Langlands's beyond endoscopy idea, a trace formula approach seems the most systematic way to get analytic continuation for all $L$-functions $L(s, \pi, \rho)$ associated to a dual group representation $\rho$ of an automorphic representation $\pi$ of a group $G$. For example, currently there is no general procedure of using integral representations to get the analytic continuation for the symmetric power $L$-functions. From the beyond endoscopy perspective, asking for the analytic continuation is certainly a more difficult question than investigating whether the $L$-function has a pole at $s=1$ or not. The question requires a deeper understanding of the geometric side of the trace formula, and this paper is just the first step in that direction.

Voronoi summation. If one can always recover the functional equation from the trace formula, then from the perspective of analytic number theory, the Voronoi summation should be implied also from the trace formula. For example in [Kowalski et al. 2000; 2002], an application of a trace formula and a Voronoi summation are used to get results on subconvexity. Could one avoid Voronoi summation and just apply the trace formula? In [Herman $\geq 2012 \mathrm{a}$ ], we do just that to get subconvexity for the Rankin-Selberg $L$-function in both levels by applying a double trace formula instead of a Voronoi summation and a single trace formula.

1.3. Key steps in proof. As for the proof of the main theorem, one sees the role of the sum over the Kloosterman sums on the geometric side of the trace formula 
interacting with the averaging coming from the Dirichlet series for the standard $L$-function.

To see the functional equation of a $\mathrm{GL}_{2} L$-function, the Dirichlet series sum exchanges roles with the sum of Kloosterman sums. There are two important steps in this switching of roles of parametrization. One is elementary reciprocity,

$$
\frac{\bar{A}}{B}+\frac{\bar{B}}{A} \equiv \frac{1}{A B}(1)
$$

which allows one to invert the modulus of exponential sums. This simple reciprocity seems to come up several times in these beyond endoscopy calculations (see [Herman 2012; $\geq 2012 b]$ ). The second important tool is the integral representation

$$
\int_{0}^{\infty} \exp (-\alpha x) J_{v}(2 \beta \sqrt{x}) J_{v}(2 \gamma \sqrt{x}) d x=\frac{1}{\alpha} I_{\nu}\left(\frac{2 \beta \gamma}{\alpha}\right) \exp \left(\frac{-\left(\beta^{2}+\gamma^{2}\right)}{\alpha}\right) d x .
$$

Given that Bessel functions are the archimedean version of Kloosterman sums, this representation implies that a Fourier transform of a product of Kloosterman sums is another Kloosterman sum times an exponential sum. It would be nice to see how these two steps are generalized for higher rank or for a relative trace formula for other groups.

\section{Preliminaries}

We recall the functional equation for a cusp form. Let $D$ be a squarefree integer, $\chi$ be a primitive Dirichlet character modulo $D$, and $k \geq 2, k \in 2 \mathbb{Z}$. Let $f \in S_{k}(D, \chi)$, where $S_{k}(D, \chi)$ is the space of holomorphic modular forms of weight $k$ and level $D$ with nebentypus $\chi$; see [Iwaniec and Kowalski 2004]. In this case the space $S_{k}(D, \chi)$ can be spanned by an orthonormal basis of primitive newforms which we label $B_{k}(D, \chi)$. We note the Fourier coefficients $c_{n}(f) n^{(k-1) / 2}$ of a form $f$ in $B_{k}(D, \chi)$ satisfy

$$
c_{n}(f) c_{l}(f)=\sum_{r \mid(n, l)} \chi(r) c_{(n l) / r^{2}}(f)
$$

for $(n l, D)=1$, and also that $\left|c_{D}(f)\right|=1$.

Let $L(f, s)=\sum_{n=1}^{\infty} c_{n}(f) / n^{s}$, and define $\Lambda(f, s)=\gamma(f, s) L(f, s)$, where

$$
\gamma(f, s)=\left(\frac{\sqrt{D}}{2 \pi}\right)^{s} \Gamma\left(\frac{s+\frac{k-1}{2}}{2}\right) \Gamma\left(\frac{s+\frac{k+1}{2}}{2}\right) .
$$

The functional equation then says $\Lambda(f, s)=i^{k} \Lambda(f, 1-s)$.

The trace formula we use is Petersson's formula, which is a variant of the relative trace formula [Knightly and Li 2006a]. This formula requires a normalization of 
the Fourier coefficients. For $c_{n}(f)$ above, define

$$
a_{n}(f):=\frac{\sqrt{\pi^{-k} \Gamma(k)}}{2^{k-1}} c_{n}(f) .
$$

Petersson's formula states

$$
\sum_{f \in B_{k}(D, \chi)} a_{n}(f) \overline{a_{l}(f)}=\delta_{n, l}+2 \pi i^{-k} \sum_{c \equiv 0(D)}^{\infty} \frac{S_{\chi}(n, l, c)}{c} J_{k-1}\left(\frac{4 \pi \sqrt{n l}}{c}\right) .
$$

Here

$$
S_{\chi}(a, b, c)=\sum_{x(c)^{*}} \overline{\chi(x)}\left(\frac{a x+b \bar{x}}{c}\right),
$$

where $x \bar{x} \equiv 1(c), e(x):=\exp (2 \pi i x)$ and $J_{t}(x)$ is the $J$-Bessel function with index $t$.

To relate the functional equation to the geometric side of the trace formula, we need an equivalent version of the functional equation for a form $f \in B_{k}(D, \chi)$, which is called Voronoi summation. The Voronoi summation needed is proved in the appendix of [Kowalski et al. 2002], and states this:

Theorem 2.1. Let $g \in C_{0}^{\infty}\left(\mathbb{R}^{+}\right)$and $f \in B_{k}(D, \chi)$, then for integers $a, c$ such that $(a D, c)=1$,

$$
\begin{aligned}
& \sum_{n \geq 1} a_{n}(f) e\left(\frac{a n}{c}\right) g(n) \\
& \quad=\frac{2 \pi i^{k} \eta(f) \chi(-c)}{c \sqrt{D}} \sum_{n \geq 1} a_{n}\left(f_{D}\right) e\left(\frac{-n \overline{a D}}{c}\right) \int_{0}^{\infty} g(x) J_{k-1}\left(\frac{4 \pi \sqrt{n x}}{\sqrt{D} c}\right) d x,
\end{aligned}
$$

where $a \bar{a} \equiv 1(c)$. Here $\eta(f)=\tau(\chi) /\left(a_{D}(f) \sqrt{D}\right)$, with $\tau(\chi)$ denoting the Gauss sum associated to $\chi$, and

$$
a_{n}\left(f_{D}\right)= \begin{cases}\overline{\chi(n)} a_{n}(f) & \text { if }(n, D)=1 \\ \overline{a_{n}(f)} & \text { if } n \mid D^{\infty}\end{cases}
$$

In our case, we only take $a=c=1$. If so, the functional equation of the $L$ function $L(f, s)$ is equivalent to the Voronoi summation by using Mellin inversion on the left hand side of (2-2), then applying the functional equation to $L(f, s)$ and using the integral representation

$$
J_{k-1}(x)=\frac{1}{4 \pi i} \int_{(\sigma)}\left(\frac{x}{2}\right)^{-s} \frac{\Gamma\left(\frac{1}{2}\left(s+\frac{k-1}{2}\right)\right)}{\Gamma\left(\frac{1}{2}\left(1-\frac{s}{2}+\frac{k-1}{2}\right)\right)} d s
$$

for $0<\sigma<1$, along with the duplication formula for the gamma function. 


\section{Main theorem}

The main theorem of the paper is:

Theorem 3.1. Let $g \in C_{0}^{\infty}\left(\mathbb{R}^{+}\right)$satisfy $\left|x^{j} g^{(j)}(x)\right| \ll(1+|\log x|)$. Then, for any $l \in \mathbb{N}$ with $(l, D)=1$, and assuming Petersson's formula above, one gets

$$
\begin{aligned}
& \sum_{f \in B_{k}(D, \chi)} \overline{a_{l}(f)} \sum_{n \geq 1} a_{n}(f) g(n) \\
& \quad=\sum_{f \in B_{k}(D, \chi)} \overline{a_{l}(f)}\left[\frac{2 \pi i^{k} \eta(f)}{\sqrt{D}} \sum_{n} a_{n}\left(f_{D}\right) \int_{0}^{\infty} g(x) J_{k-1}\left(\frac{4 \pi \sqrt{n x}}{\sqrt{D}}\right) d x\right] .
\end{aligned}
$$

Using Hecke theory one gets:

Corollary 3.2. For a modular form $f \in B_{k}(D, \chi)$,

$$
L(f, s)=\frac{i^{k} \gamma(f, 1-s) L(f, 1-s)}{\gamma(f, s)},
$$

or,

$$
\Lambda(f, s)=\Lambda(f, 1-s) .
$$

Proof of Theorem 3.1. Using Petersson's trace formula on the left hand side of (3-1) one gets

$$
\begin{aligned}
\sum_{n} g(n)\left[\delta_{n, l}+2 \pi i^{-k} \sum_{c=1}^{\infty} \frac{S_{\chi}(n, l, D c)}{D c} J_{k-1}\left(\frac{4 \pi \sqrt{n l}}{D c}\right)\right] \\
=g(l)+2 \pi i^{-k} \sum_{c=1}^{\infty} \sum_{n} g(n) \frac{S_{\chi}(n, l, D c)}{D c} J_{k-1}\left(\frac{4 \pi \sqrt{n l}}{D c}\right) .
\end{aligned}
$$

We can interchange the $c$-sum and $n$-sum as the latter is compactly supported.

For now we will ignore the term $g(l)$, and come back to it later. Opening up the Kloosterman sum and gathering the $n$-sum together, we apply Poisson summation on it in arithmetic progressions modulo $c$, getting

$$
\begin{aligned}
2 \pi i^{-k} \sum_{c=1}^{\infty} \frac{1}{(D c)^{2}} \sum_{x(D c)^{*}} \chi(x) e\left(\frac{\bar{x} l}{D c}\right) \sum_{m \in \mathbb{Z}} & \sum_{k(D c)} e\left(\frac{x k+m k}{D c}\right) \\
& \times \int_{-\infty}^{\infty} g(t) J_{k-1}\left(\frac{4 \pi \sqrt{t l}}{D c}\right) e\left(\frac{-m t}{D c}\right) d t .
\end{aligned}
$$

Using

$$
\sum_{a(c)} e\left(\frac{a x}{c}\right)= \begin{cases}c & \text { if } x \equiv 0(c), \\ 0 & \text { else }\end{cases}
$$


one gets

$$
2 \pi i^{-k} \sum_{c=1}^{\infty} \frac{1}{D c} \sum_{\substack{m \neq 0 \in \mathbb{Z} \\(m, D c)=1}} \overline{\chi(m)} e\left(\frac{-l \bar{m}}{D c}\right) \int_{-\infty}^{\infty} g(t) J_{k-1}\left(\frac{4 \pi \sqrt{t l}}{D c}\right) e\left(\frac{-m t}{D c}\right) d t
$$

Note the $m=0$ disappears.

Now the interesting part of the argument is that the $c$-sum and $n$-sum swap roles, in that the $c$-sum will become part of the averaging coming from the $L$-function.

We use the elementary reciprocity

$$
\frac{\bar{A}}{B}+\frac{\bar{B}}{A} \equiv \frac{1}{A B}(1),
$$

to get

$$
\begin{aligned}
2 \pi i^{-k} \sum_{c=1}^{\infty} \frac{1}{D c} \sum_{\substack{m \neq 0 \in \mathbb{Z} \\
(m, D c)=1}} \overline{\chi(m)} e\left(\frac{l \bar{c}}{D m}\right) & e\left(\frac{-l}{m D c}\right) \\
& \times \int_{-\infty}^{\infty} g(t) J_{k-1}\left(\frac{4 \pi \sqrt{t l}}{D c}\right) e\left(\frac{-m t}{D c}\right) d t .
\end{aligned}
$$

Also, the terms $m<0$ we write as $-m, m \in \mathbb{N}$, and exchange sign to the $c$-sum. This can be clearly done everywhere except for in the $J$-Bessel function and the $\frac{1}{c}$ term. Using the fact that $J_{k-1}(-x)=-J_{k-1}(x)$, we can rewrite (3-5) as

$$
\begin{aligned}
2 \pi i^{-k} \sum_{c \neq 0, c \in \mathbb{Z}}^{\infty} \frac{1}{D c} \sum_{\substack{m=1 \\
(m, c)=1}} \overline{\chi(m)} e\left(\frac{l \bar{c}}{D m}\right) & e\left(\frac{-l}{m D c}\right) \\
& \times \int_{-\infty}^{\infty} g(t) J_{k-1}\left(\frac{4 \pi \sqrt{t l}}{D c}\right) e\left(\frac{-m t}{D c}\right) d t .
\end{aligned}
$$

The rearrangement of the $m$-sum is accomplished by using a standard integration by parts argument in the $t$-integral and the estimate in the appendix of [Kowalski et al. 2002],

$$
\left|z^{k} J_{v}(z)\right| \ll_{k, v} \frac{1}{(1+z)^{1 / 2}}
$$

for $\mathfrak{R} v \geq 0$.

We also interchange the $c$-sum and $m$-sum. To justify the rearrangement, note for $c$ large, and by using the power series expansion, we have the estimate

$$
J_{k-1}\left(\frac{4 \pi \sqrt{t l}}{D c}\right) \ll \frac{1}{c^{k-1}} .
$$


Therefore, for $N$ sufficiently large, by estimating the exponentials and integral trivially and noting that $k \geq 2$, we get

$$
\begin{aligned}
\sum_{c>N} \frac{1}{D c} \sum_{\substack{m=1 \\
(m, c)=1}} \overline{\chi(m)} e\left(\frac{l \bar{c}}{D m}\right) e\left(\frac{-l}{m D c}\right) \int_{-\infty}^{\infty} g(t) J_{k-1}\left(\frac{4 \pi \sqrt{t l}}{D c}\right) e\left(\frac{-m t}{D c}\right) d t \\
\ll L(0, \bar{\chi}) \sum_{c>N} \frac{1}{c^{k}}<\infty .
\end{aligned}
$$

Clearly, the $c$-sum up to $N$ is finite and is not a problem, and the sums can be interchanged.

Now we need a integral representation from [Gradshteyn and Ryzhik 2000, $6.615]$,

$$
\int_{0}^{\infty} \exp (-\alpha x) J_{v}(2 \beta \sqrt{x}) J_{v}(2 \gamma \sqrt{x}) d x=\frac{1}{\alpha} I_{v}\left(\frac{2 \beta \gamma}{\alpha}\right) \exp \left(\frac{-\left(\beta^{2}+\gamma^{2}\right)}{\alpha}\right)
$$

for $\Re \nu>-1$.

We rewrite (3-6) as

$$
\begin{aligned}
(2 \pi i)\left(2 \pi i^{-k}\right) \sum_{m} \frac{1}{m} \sum_{\substack{c \neq 0, c \in Z \\
(c, m)=1}} \overline{\chi(m)} e\left(\frac{l \bar{c}}{D m}\right) & \\
& \times \int_{-\infty}^{\infty} g(t)\left[\frac{m}{2 \pi i D c} J_{k-1}\left(\frac{4 \pi \sqrt{t l}}{D c}\right) e\left(\frac{-l}{m D c}\right) e\left(\frac{-m t}{D c}\right)\right] d t .
\end{aligned}
$$

Note the term in brackets is equal to the right hand side of (3-8) times $i^{k-1}$ for $\alpha=2 \pi i D c / m, \beta=2 \pi \sqrt{l} / m$, and $\gamma=2 \pi \sqrt{t}$ by using the fact that for $k-1$ odd, $J_{k-1}(z)=i^{k-1} I_{k-1}(-i y)$.

Using this integral representation, one has

$$
\begin{aligned}
4 \pi^{2} \sum_{m} & \frac{1}{m} \sum_{\substack{c \neq 0, c \in Z \\
(c, m)=1}} \overline{\chi(m)} e\left(\frac{l \bar{c}}{D m}\right) \\
& \times \int_{-\infty}^{\infty} g(t)\left[\int_{0}^{\infty} J_{k-1}\left(\frac{4 \pi \sqrt{l y}}{m}\right) J_{k-1}(4 \pi \sqrt{t y}) e\left(\frac{-D c y}{m}\right) d y\right] d t .
\end{aligned}
$$

We make a change of variables $y \rightarrow y / D$ to get

$$
\begin{aligned}
& 4 \pi^{2} \sum_{m} \frac{1}{D m} \sum_{\substack{c \neq 0, c \in Z \\
(c, m)=1}} \overline{\chi(m)} e\left(\frac{l \bar{c}}{D m}\right) \\
& \quad \times \int_{-\infty}^{\infty} g(t)\left[\int_{0}^{\infty} J_{k-1}\left(\frac{4 \pi \sqrt{l D y}}{D m}\right) J_{k-1}\left(\frac{4 \pi \sqrt{t y}}{\sqrt{D}}\right) e\left(\frac{-c y}{m}\right) d y\right] d t .
\end{aligned}
$$


Using $\tau(\chi) \tau(\bar{\chi}) / D=1$, we get

$$
\begin{aligned}
& \frac{4 \pi^{2} \tau(\chi)}{D} \sum_{m=1} \frac{1}{D m} \sum_{c \in \mathbb{Z}} \overline{\chi(m)} \tau(\bar{\chi}) e\left(\frac{l \bar{c}}{D m}\right) \\
& \quad \times \int_{-\infty}^{\infty} J_{k-1}\left(\frac{4 \pi \sqrt{l D y}}{D m}\right)\left[\int_{0}^{\infty} g(t) J_{k-1}\left(\frac{4 \pi \sqrt{t y}}{\sqrt{D}}\right) d t\right] e\left(\frac{-c y}{m}\right) d y
\end{aligned}
$$

Anticipating the use of the Chinese remainder theorem, we let $c^{\prime}=D c$. So (3-11) equals

$$
\begin{aligned}
& \frac{4 \pi^{2} \tau(\chi)}{D} \sum_{m=1} \frac{1}{D m} \sum_{\begin{array}{c}
c^{\prime} \in \mathbb{Z}, c^{\prime} \equiv 0(D) \\
c^{\prime} \neq 0,\left(c^{\prime}, m\right)=1
\end{array}} \overline{\chi(m)} \tau(\bar{\chi}) e\left(\frac{l \overline{c^{\prime} / D}}{D m}\right) \\
& \quad \times \int_{-\infty}^{\infty} J_{k-1}\left(\frac{4 \pi \sqrt{l D y}}{D m}\right)\left[\int_{0}^{\infty} g(t) J_{k-1}\left(\frac{4 \pi \sqrt{t y}}{\sqrt{D}}\right) d t\right] e\left(\frac{-c^{\prime} y}{D m}\right) d y .
\end{aligned}
$$

We focus on the arithmetic inside the $c^{\prime}$-sum. We note, using $(m, D)=1$ and the Chinese remainder theorem, that

$$
\begin{aligned}
\overline{\chi(m)} \tau(\bar{\chi}) e\left(\frac{l \overline{c^{\prime} / D}}{D m}\right) & =\left[\sum_{a(D)} \bar{\chi}(a) e\left(\frac{\bar{m} a}{D}\right)\right]\left[\sum_{b(m)^{*}, \bar{b} l \equiv \frac{c^{\prime}}{D}(m)} e\left(\frac{b}{m}\right)\right] \\
& =\sum_{\substack{\chi(D m) \\
D \bar{x} l \equiv c^{\prime}(D m)}} \overline{\chi(x)} e\left(\frac{x}{D m}\right) .
\end{aligned}
$$

Using (3-3) again the above equals

$$
\sum_{\substack{x(D m) \\ D \bar{x} l \equiv c^{\prime}(D m)}} \overline{\chi(x)} e\left(\frac{x}{D m}\right)=\frac{1}{D m} \sum_{x(D m)} \overline{\chi(x)} e\left(\frac{x}{D m}\right) \sum_{k(D m)} e\left(\frac{k\left(D l \bar{x}-c^{\prime}\right)}{D m}\right) .
$$

Incorporating the above line and a rearrangement of the exponential sums, we have

$$
\begin{aligned}
& \frac{4 \pi^{2} \tau(\chi)}{D} \sum_{m=1} \frac{1}{(D m)^{2}} \sum_{c^{\prime} \in \mathbb{Z}} \sum_{x(D m)} \overline{\chi(x)} e\left(\frac{x}{D m}\right) \sum_{k(D m)} e\left(\frac{k D l \bar{x}}{D m}\right) \\
& \times \int_{0}^{\infty} J_{k-1}\left(\frac{4 \pi \sqrt{l D y}}{D m}\right)\left[\int_{0}^{\infty} g(t) J_{k-1}\left(\frac{4 \pi \sqrt{t y}}{\sqrt{D}}\right) d t\right] e\left(\frac{-c^{\prime}(y+k)}{D m}\right) d y
\end{aligned}
$$


We note the $c^{\prime}$-sum has the restriction $c^{\prime} \equiv 0(D)$ removed by the $k$-sum. With a change of variables $y \rightarrow y-k$, followed by $y \rightarrow D m y$, we get

$$
\begin{aligned}
& \frac{4 \pi^{2} \tau(\chi)}{D} \sum_{m=1} \frac{1}{D m} \sum_{x(D m)^{*}} \overline{\chi(x)} e\left(\frac{x}{D m}\right) \sum_{k(D m)} e\left(\frac{k D l \bar{x}}{D m}\right) \\
&+\sum_{c^{\prime} \in \mathbb{Z}} \int_{0}^{\infty} J_{k-1}\left(\frac{4 \pi \sqrt{l D(D m y-k)}}{D m}\right) \\
& \times\left[\int_{0}^{\infty} g(t) J_{k-1}\left(\frac{4 \pi \sqrt{t(D m y-k)}}{\sqrt{D}}\right) d t\right] e\left(-c^{\prime} y\right) d y .
\end{aligned}
$$

The $c^{\prime}$-sum now clearly came from a Poisson summation, namely,

$$
\begin{aligned}
& \sum_{c^{\prime} \in \mathbb{Z}} \int_{0}^{\infty} J_{k-1}\left(\frac{4 \pi \sqrt{l D(D m y-k)}}{D m}\right) \\
& \times\left[\int_{0}^{\infty} g(t) J_{k-1}\left(\frac{4 \pi \sqrt{t(D m y-k)}}{\sqrt{D}}\right) d t\right] e\left(-c^{\prime} y\right) d y \\
&=\sum_{c \in \mathbb{Z}} J_{k-1}\left(\frac{4 \pi \sqrt{l D\left(D m c^{\prime}-k\right)}}{D m}\right) \\
& \times \int_{0}^{\infty} g(t) J_{k-1}\left(\frac{4 \pi \sqrt{t(D m c-k)}}{\sqrt{D}}\right) d t .
\end{aligned}
$$

In order to check that

$$
F(w)=J_{k-1}\left(\frac{4 \pi \sqrt{l D(D m w-k)}}{D m}\right) \int_{0}^{\infty} g(t) J_{k-1}\left(\frac{4 \pi \sqrt{t(D m w-k)}}{D}\right) d t
$$

satisfies the conditions for Poisson summation, we use the following lemma of [Kowalski et al. 2002]:

Lemma 3.3. Let $h(x)$ be a smooth function supported on $[M, 2 M]$ that satisfies $\left|x^{j} h^{(j)}(x)\right| \ll(1+|\log x|)$ for all $i \geq 0, x>0$. For $v$ complex and $j \geq 0$ we have

$$
\int_{0}^{\infty} J_{v}(x) h(x) d x \ll_{v, j} \frac{(1+|\log M|)}{M^{j-1}} \frac{M^{\Re v+j+1}}{(1+M)^{\Re v+j+1 / 2}} .
$$

We apply this to the integral in $F(w)$ with

$$
h(t)=\frac{D^{2}}{16 \pi^{2}(D m w-k)^{2}} \operatorname{tg}\left(\frac{D^{2} t^{2}}{16 \pi^{2}(D m w-k)^{2}}\right) .
$$

It is easy, but tedious, to check that the assumptions of the lemma are fulfilled by using the assumption on $g$ that $\left|x^{j} g^{(j)}(x)\right| \ll(1+|\log x|)$ (from the hypothesis of Theorem 3.1). The lemma then gives $F(w) \ll \min \left(w^{k-1}, 1 / w^{j}\right)$ for any $j>0$ for $w \in[0, \infty)$. So certainly Poisson summation holds in this case. 
Defining $D m c-k=-j,(3-15)$ again by regrouping equals

$$
\begin{aligned}
& \frac{4 \pi^{2} \tau(\chi)}{D} \sum_{m=1} \frac{1}{D m} \sum_{j \in \mathbb{Z}} \sum_{x(D m)^{*}} \overline{\chi(x)} e\left(\frac{x}{D m}\right) e\left(\frac{j D l \bar{x}}{D m}\right) J_{k-1}\left(\frac{4 \pi \sqrt{l j D}}{D m}\right) \\
& \times \int_{0}^{\infty} g(t) J_{k-1}\left(\frac{4 \pi \sqrt{t j}}{\sqrt{D}}\right) d t \\
& =\frac{4 \pi^{2} \tau(\chi)}{D} \sum_{m=1} \frac{1}{D m} \sum_{j \in \mathbb{Z}} \overline{\chi(j)} S_{\chi}(D l, j, D m) J_{k-1}\left(\frac{4 \pi \sqrt{l D j}}{D m}\right) \\
& \times \int_{0}^{\infty} g(t) J_{k-1}\left(\frac{4 \pi \sqrt{t j}}{\sqrt{D}}\right) d t \\
& =\frac{4 \pi^{2} \tau(\chi)}{D} \sum_{j \in \mathbb{Z}} \overline{\chi(j)}\left[\sum_{m \equiv 0(D)} \frac{S_{\chi}(D l, j, m)}{m} J_{k-1}\left(\frac{4 \pi \sqrt{D l j}}{m}\right)\right] \\
& \times \int_{0}^{\infty} g(t) J_{k-1}\left(\frac{4 \pi \sqrt{t j}}{\sqrt{D}}\right) d t
\end{aligned}
$$

Now recall we ignored $g(l)$ from (3-2), so (3-1) equals

$$
\begin{array}{r}
g(l)+\frac{2 \pi i^{k} \tau(\chi)}{D} \sum_{j} \overline{\chi(j)}\left[2 \pi i^{-k} \sum_{m \equiv 0(D)} \frac{S_{\chi}(D l, j, m)}{m} J_{k-1}\left(\frac{4 \pi \sqrt{D l j}}{m}\right)\right] \\
\times \int_{0}^{\infty} g(t) J_{k-1}\left(\frac{4 \pi \sqrt{t j}}{\sqrt{D}}\right) d t
\end{array}
$$

The $g(l)$ term is again the diagonal term for the geometric side of the trace formula that comes from the term

$$
\sum_{f_{D}} a_{l D}\left(f_{D}\right) \overline{a_{l D}\left(f_{D}\right)}
$$

This is again using the fact that $\left|a_{D}\left(f_{D}\right)\right|=1$.

Now as $D$ is squarefree and $\chi$ is primitive, the space $B_{k}(N, \chi)$ is spanned by newforms, which implies the Fourier coefficients are multiplicative in all the primes (including the bad primes) and $\left|c_{D}(f)\right|=1$. So using Petersson's formula again we get

$$
\begin{aligned}
& \sum_{f \in B_{k}} \overline{a_{l}(f)}\left[\frac{2 \pi i^{k} \tau(\chi)}{D a_{D}(f)} \sum_{j} \overline{\chi(j)} a_{j}(f) \int_{0}^{\infty} g(x) J_{k-1}\left(\frac{4 \pi \sqrt{j x}}{\sqrt{D}}\right) d x\right] \\
& =\sum_{f \in B_{k}} \overline{a_{l}(f)}\left[\frac{2 \pi i^{k} \eta(f)}{\sqrt{D}} \sum_{j} \overline{\chi(j)} a_{j}(f) \int_{0}^{\infty} g(x) J_{k-1}\left(\frac{4 \pi \sqrt{j x}}{\sqrt{D}}\right) d x\right] \\
& =\sum_{f_{D} \in B_{k}} \overline{a_{l}\left(f_{D}\right)}\left[\frac{2 \pi i^{k} \eta(f)}{\sqrt{D}} \sum_{(j, D)=1} a_{j}\left(f_{D}\right) \int_{0}^{\infty} g(x) J_{k-1}\left(\frac{4 \pi \sqrt{j x}}{\sqrt{D}}\right) d x\right] .
\end{aligned}
$$


Note that to show the connection to Voronoi summation from Theorem 2.1, we need also the coefficients $a_{j}\left(f_{D}\right)$ with $(j, D)>1$. We state a lemma:

Lemma 3.4. For $(l, D)=1$,

$$
\sum_{f_{D} \in B_{k}} \overline{a_{l}\left(f_{D}\right)}\left[\frac{2 \pi i^{k} \eta(f)}{\sqrt{D}} \sum_{(j, D)>1} a_{j}\left(f_{D}\right) \int_{0}^{\infty} g(x) J_{k-1}\left(\frac{4 \pi \sqrt{j x}}{\sqrt{D}}\right) d x\right]=0 .
$$

Assuming the lemma for now, we get that (3-19) equals

$$
\sum_{f_{D} \in B_{k}} \overline{a_{l}\left(f_{D}\right)}\left[\frac{2 \pi i^{k} \eta(f)}{\sqrt{D}} \sum_{j=1}^{\infty} a_{j}\left(f_{D}\right) \int_{0}^{\infty} g(x) J_{k-1}\left(\frac{4 \pi \sqrt{j x}}{\sqrt{D}}\right) d x\right],
$$

which proves Theorem 3.1.

Proof of Lemma 3.4. First we write $j=D^{k} j^{\prime},\left(j^{\prime}, D\right)=1$. Using the definition of the coefficients $f_{D}(n)$ in Theorem 2.1, the left hand side of (3-20) equals

$$
\frac{2 \pi i^{k}}{\sqrt{D}} \sum_{k=1}^{\infty} \sum_{(j, D)=1} \overline{\chi(j)} \sum_{f \in B_{k}} \overline{a_{l D^{k+1}}(f)} a_{j}(f) \int_{0}^{\infty} g(x) J_{k-1}\left(\frac{4 \pi \sqrt{j D^{k} x}}{\sqrt{D}}\right) d x .
$$

Fix a $k$, and following the same argument as we made previously, we apply Petersson's formula to get

$$
\begin{aligned}
2 \pi i^{-k} \sum_{j} \overline{\chi(j)} \sum_{c=1}^{\infty} \frac{S_{\chi}\left(j, l D^{k+1}, D c\right)}{D c} J_{k-1}\left(\frac{4 \pi \sqrt{n l D^{k+1}}}{D c}\right) \\
\quad \times \int_{0}^{\infty} g(x) J_{k-1}\left(\frac{4 \pi \sqrt{j D^{k} x}}{\sqrt{D}}\right) d x .
\end{aligned}
$$

That we can apply Petersson's formula in this case follows from using the estimates of Lemma 3.3. With a change of variable in the Kloosterman sum this equals

$$
\begin{aligned}
2 \pi i^{-k} \sum_{j} \sum_{c=1}^{\infty} \frac{S_{\chi}\left(1, j l D^{k+1}, D c\right)}{D c} J_{k-1}\left(\frac{4 \pi \sqrt{n l D^{k+1}}}{D c}\right) & \\
& \times \int_{0}^{\infty} g(x) J_{k-1}\left(\frac{4 \pi \sqrt{j D^{k} x}}{\sqrt{D}}\right) d x .
\end{aligned}
$$

Interchanging the $j$ - and $c$-sums, justified by a similar Bessel function analysis as above, we apply Poisson summation to the $j$-sum modulo $D c$. The crucial arithmetic sums, analogous to the ones in obtaining (3-4), are

$$
\sum_{x(D c)^{*}} \overline{\chi(x)} e\left(\frac{x}{D c}\right) \sum_{a(D c)} e\left(\frac{\bar{x} a D^{k+1} l}{D c}\right) e\left(\frac{-a m}{D c}\right),
$$


where $m$ is the variable for Poisson summation. The inner sum is nonzero only when $D^{k+1} l \equiv m x(D c)$. If $(m, c)=1$, then it is easy to check (3-21) is zero. As well if $D^{h} \mid m$ then $D^{h-1} \mid c$ as $(x l, D)=1$ for $h \leq k+1$. So for a nonzero contribution we must have $D^{k+1} \mid m$ and $D^{k} \mid c$. Writing $c=D^{k} c^{\prime}$ and $m=D^{k+1} m^{\prime}$, $x$ must satisfy $l \equiv m^{\prime} x\left(c^{\prime}\right)$. We can write these solutions as $x \equiv \bar{m}^{\prime} l+c^{\prime} b(D c)$, where $b\left(D^{k+1}\right)$. So (3-21) equals

$$
D c \sum_{b\left(D^{k+1}\right)} \bar{\chi}\left(\bar{m}^{\prime} l+c^{\prime} b\right) e\left(\frac{\bar{m}^{\prime} l+c^{\prime} b}{D c}\right)=e\left(\frac{\bar{m}^{\prime} l}{D c}\right) D c \sum_{b\left(D^{k+1}\right)} \bar{\chi}\left(\bar{m}^{\prime} l+c^{\prime} b\right) e\left(\frac{b}{D^{k+1}}\right) .
$$

With a change of variables $b \rightarrow \bar{c} b, b \rightarrow b-\bar{m}^{\prime} l$, the inner Gauss sum is

$$
\sum_{b\left(D^{k+1}\right)} \bar{\chi}(b) e\left(\frac{\bar{c}^{\prime} b}{D^{k+1}}\right)=\overline{\chi\left(c^{\prime}\right)} \sum_{b\left(D^{k+1}\right)} \bar{\chi}(b) e\left(\frac{b}{D^{k+1}}\right) .
$$

This last Gauss sum is zero as $\chi$ is a primitive character modulo $D$ and $k+1 \geq 2$.

Remark. There is nothing special about the test function we used in the lemma, and by a similar argument it is easy to show that for a "nice" test function $V(x)$ and $k \geq 2$,

$$
\sum_{j=1}^{\infty} \overline{\chi(j)} V(j) \sum_{f \in B_{k}} \overline{a_{D^{k}}(f)} a_{j}(f)=0 .
$$

\section{Application of Hecke theory}

Now to prove Corollary 3.2. One can rewrite Theorem 3.1 as

(4-1) $\frac{1}{2 \pi i} \int_{(\sigma)} G(s)\left[\sum_{f \in B_{k}} \overline{a_{l}(f)}\left(L(f, s)-\frac{i^{k} \gamma(f, 1-s) L(f, 1-s)}{\gamma(f, s)}\right)\right] d s=0$,

using that the Voronoi summation we take into consideration is equivalent to the functional equation. Since (4-1) holds for any $g$ in $C_{0}^{\infty}\left(\mathbb{R}^{+}\right)$, and in fact holds with slightly more care for the transform of any Schwarz function, by completeness, it must hold that

$$
\sum_{f \in B_{k}} \overline{a_{l}(f)}\left(L(f, s)-\frac{i^{k} \gamma(f, 1-s) L(f, 1-s)}{\gamma(f, s)}\right)=0 .
$$

Fix a form $f^{\circ} \in B_{k}$. Now as $l$ was arbitrary and the space of forms $f \in B_{k}$ is finite dimensional, using the relation

$$
a_{n}(f) a_{l}(f)=\sum_{r \mid(n, l)} \chi(r) a_{n l / r^{2}}(f)
$$


for $(n l, D)=1$, one can build a polynomial in the Hecke coefficients, call it $F\left(a_{q_{1}}(f), a_{q_{2}}(f), \ldots, a_{q_{N}}(f)\right)$, such that

$$
\sum_{f \in B_{k}} F\left(a_{q_{1}}(f), a_{q_{2}}(f), \ldots, a_{q_{N}}(f)\right)\left(L(f, s)-\frac{i^{k} \gamma(f, 1-s) L(f, 1-s)}{\gamma(f, s)}\right)=0,
$$

where $F$ equals 1 for $f=f^{\circ}$, and equals 0 for $f \neq f^{\circ}$, following [Herman 2010]. So we get a pointwise equality

$$
L(f, s)-\frac{i^{k} \gamma(f, 1-s) L(f, 1-s)}{\gamma(f, s)}=0,
$$

which proves the corollary.

\section{Appendix}

We replicate Sarnak's argument [2001] from his letter to Langlands. In order to do so, we use the Kuznetsov trace formula for the entire $\mathrm{GL}_{2}$ spectrum. We refer to [Herman 2011] for the details. Let $H(D, \chi)$ denote the $\mathrm{GL}_{2}$ spectrum with level $D$ and nebentypus $\chi$.

Theorem A.1. Let $g, V \in C_{0}^{\infty}\left(\mathbb{R}^{+}\right)$with $\left|x^{j} g^{(j)}(x)\right| \ll(1+|\log x|)$, X a large fixed real number, and $D$ and $\chi$ as above. Then for any integer $A>0$,

$$
\sum_{n \leq X} \sum_{f \in H(D, \chi)} h\left(t_{f}, V\right) \overline{a_{l}(f)} a_{n}(f) g(n / X)=O\left(X^{-A}\right) .
$$

Proof. We apply the Kuznetsov trace formula and Poisson summation, similarly as we did in obtaining (3-4) for just the holomorphic forms, to get

$$
\sum_{c=1}^{\infty} \frac{1}{D c} \sum_{\substack{m \neq 0 \in \mathbb{Z} \\(m, D c)=1}} \overline{\chi(m)} e\left(\frac{-l \bar{m}}{D c}\right) \int_{-\infty}^{\infty} g\left(\frac{t}{X}\right) V\left(\frac{4 \pi \sqrt{t l}}{D c}\right) e\left(\frac{-m t}{D c}\right) d t .
$$

Essentially, the argument only depends on showing the integral is bounded by $O\left(X^{-A}\right)$. Note that as $V$ and $g$ are compactly supported, the $c$-sum is restricted to size $a \sqrt{X} \leq c \leq b \sqrt{X}$, for some absolute constants $a, b \in \mathbb{R}^{+}$, notated $c \sim \sqrt{X}$. Note that $g^{(k)}(D c t / X) \ll 1 / X^{k / 2}$ and $V^{(h)}(4 \pi \sqrt{t l} / \sqrt{D c}) \ll 1 / X^{h / 2}$ for $h, k \geq 0$. Also the size of the integral is $X / c \sim \sqrt{X}$. Using these estimates and integrating by parts $j$-times, after a change of variables $t \rightarrow D c t$, it easy to check that

(A-3) $D c \int_{-\infty}^{\infty} g\left(\frac{D c t}{X}\right) V\left(\frac{4 \pi \sqrt{t l}}{\sqrt{D c}}\right) e(-m t) d t \ll \frac{D c}{(\sqrt{X})^{j-1} m^{j}} \ll \frac{1}{(\sqrt{X})^{j-2} m^{j}}$. 
So including the $c$ - and $m$-sums we have

$$
\ll \frac{1}{(\sqrt{X})^{j-2}} \sum_{c \sim \sqrt{X}} \sum_{m} \frac{1}{m^{j}} \ll \frac{1}{(\sqrt{X})^{j-3}} .
$$

Obviously, this implies the theorem by taking $(j-3 / 2)>A$.

\section{Acknowledgements}

I want to dedicate this paper to my advisor, Jon Rogawski. I could not have asked for a better advisor than Jon as a graduate student at UCLA. His unfaltering patience and calm resolve balanced my personality, which was the opposite of patient in those days. When I would fail to understand an aspect of automorphic forms or the trace formula, Jon would dismiss my frustration and clarify the misunderstanding in a way that only Jon could with sympathetic composure and the knowledge of a veteran in the field. While he was always collected when he addressed the challenges that I faced, he was a passionate person and was ardent when he spoke about math. During times of stagnation, I would go to Jon to reinvigorate me. After talking to Jon, I always felt more inspired and confident.

From winter to the early part of summer of 2011, I was at the American Institute of Mathematics in Palo Alto and would visit Jon in Los Angeles every few months. I remember fondly going to coffee shops or to his home to discuss my new ideas as he shared his own. It was in one of these gatherings that he suggested how to isolate a single Hecke eigenform (found in Section 4). I want to point this out because even today, Jon continues to inspire me. I am honored to have been one of his students and am also saddened that I was his last.

\section{References}

[Frenkel et al. 2010] E. Frenkel, R. Langlands, and B. C. Ngô, "Formule des traces et fonctorialité: le début d'un programme", Ann. Sci. Math. Québec 34:2 (2010), 199-243. MR 2012c:11240 Zbl 05998868 arXiv 1003.4578

[Godement and Jacquet 1972] R. Godement and H. Jacquet, Zeta functions of simple algebras, Lecture Notes in Mathematics 260, Springer, Berlin, 1972. MR 49 \#7241 Zbl 0244.12011

[Gradshteyn and Ryzhik 2000] I. S. Gradshteyn and I. M. Ryzhik, Table of integrals, series, and products, 6th ed., Academic Press, San Diego, CA, 2000. MR 2001c:00002 Zbl 0981.65001

[Hecke 1918] E. Hecke, "Eine neue Art von Zetafunktionen und ihre Beziehungen zur Verteilung der Primzahlen”, Math. Z. 1:4 (1918), 357-376. MR 1544302 JFM 46.0258.01

[Hecke 1920] E. Hecke, "Eine neue Art von Zetafunktionen und ihre Beziehungen zur Verteilung der Primzahlen”, Math. Z. 6:1-2 (1920), 11-51. MR 1544392

[Herman 2010] P. E. Herman, "Quadratic base change and the analytic continuation of the Asai L-function: a new trace formula approach”, preprint, 2010. arXiv 1008.3921

[Herman 2011] P. E. Herman, "Beyond endoscopy for the Rankin-Selberg L-function", J. Number Theory 131:9 (2011), 1691-1722. MR 2802142 Zbl 1245.11068 
[Herman 2012] P. E. Herman, "Beyond endoscopy for the symmetric cube $L$-function and the Shimura correspondence”, preprint, 2012. arXiv 1208.1705

[Herman $\geq 2012$ a] P. E. Herman, "Subconvexity for the Rankin-Selberg $L$-function in both levels", in preparation.

[Herman $\geq 2012$ b] P. E. Herman, "A trace formula approach to $S y m^{2} \otimes S y m^{2}$ ", in preparation.

[Iwaniec 1984] H. Iwaniec, "Prime geodesic theorem", J. Reine Angew. Math. 349 (1984), 136-159. MR 85h:11025 Zbl 0527.10021

[Iwaniec and Kowalski 2004] H. Iwaniec and E. Kowalski, Analytic number theory, American Mathematical Society Colloquium Publications 53, American Mathematical Society, Providence, RI, 2004. MR 2005h:11005 Zbl 1059.11001

[Jacquet and Langlands 1970] H. Jacquet and R. P. Langlands, Automorphic forms on GL(2), Lecture Notes in Mathematics 114, Springer, Berlin, 1970. MR 53 \#5481 Zbl 0236.12010

[Knightly and Li 2006a] A. Knightly and C. Li, "A relative trace formula proof of the Petersson trace formula", Acta Arith. 122:3 (2006), 297-313. MR 2007d:11042 Zbl 1095.11028

[Knightly and Li 2006b] A. Knightly and C. Li, Traces of Hecke operators, Mathematical Surveys and Monographs 133, American Mathematical Society, Providence, RI, 2006. MR 2008g:11090 Zbl 1120.11024

[Kowalski et al. 2000] E. Kowalski, P. Michel, and J. VanderKam, "Mollification of the fourth moment of automorphic $L$-functions and arithmetic applications", Invent. Math. 142:1 (2000), 95-151. MR 2001m:11080 Zbl 1054.11026

[Kowalski et al. 2002] E. Kowalski, P. Michel, and J. VanderKam, "Rankin-Selberg $L$-functions in the level aspect”, Duke Math. J. 114:1 (2002), 123-191. MR 2004c:11070 Zbl 1035.11018

[Ngô 2010] B. C. Ngô, "Le lemme fondamental pour les algèbres de Lie", Publ. Math. Inst. Hautes Études Sci. 111 (2010), 1-169. MR 2011h:22011 Zbl 1200.22011 arXiv 0801.0446

[Rogawski 1994] J. Rogawski, "Appendix: modular forms, the Ramanujan conjecture and the Jacquet-Langlands correspondence”, pp. 135-176 in Discrete groups, expanding graphs and invariant measures, Progress in Mathematics 125, Birkhäuser, Basel, 1994. MR 96g:22018 Zbl 0826.22012

[Sarnak 2001] P. C. Sarnak, "Comments on Langland's lecture", Princeton University, 2001, available at http://web.math.princeton.edu/sarnak/SarnakLectureNotes-1.pdf.

[Tate 1967] J. T. Tate, "Fourier analysis in number fields, and Hecke's zeta-functions", pp. 305-347 in Algebraic number theory (Brighton, 1965), edited by J. W. S. Cassels and A. Fröhlich, Academic Press, London, 1967. MR 36 \#121

Received August 1, 2012.

P. EDWARD HERMAN

DEPARTMENT OF MATHEMATICS

UNIVERSITY OF CHICAGO

5734 S. UNIVERSITY AVENUE

ChICAgo, IL 60637

UNITED STATES

peherman@math.uchicago.edu 


\title{
PACIFIC JOURNAL OF MATHEMATICS
}

\author{
http://pacificmath.org
}

Founded in 1951 by E. F. Beckenbach (1906-1982) and F. Wolf (1904-1989)

\section{EDITORS}

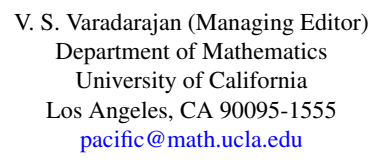

Don Blasius

Department of Mathematics University of California

Los Angeles, CA 90095-1555

blasius@math.ucla.edu

Robert Finn

Department of Mathematics

Stanford University

Stanford, CA 94305-2125

finn@math.stanford.edu

Alexander Merkurjev

Department of Mathematics

University of California

Los Angeles, CA 90095-1555

merkurev@math.ucla.edu

\author{
Vyjayanthi Chari \\ Department of Mathematics \\ University of California \\ Riverside, CA 92521-0135 \\ chari@math.ucr.edu \\ Kefeng Liu \\ Department of Mathematics \\ University of California \\ Los Angeles, CA 90095-1555 \\ liu@math.ucla.edu \\ Sorin Popa \\ Department of Mathematics \\ University of California \\ Los Angeles, CA 90095-1555 \\ popa@math.ucla.edu \\ Paul Yang \\ Department of Mathematics \\ Princeton University \\ Princeton NJ 08544-1000 \\ yang@math.princeton.edu
}

\section{PRODUCTION}

Silvio Levy, Scientific Editor, pacific@math.berkeley.edu

\section{SUPPORTING INSTITUTIONS}

ACADEMIA SINICA, TAIPEI

CALIFORNIA INST. OF TECHNOLOGY

INST. DE MATEMÁTICA PURA E APLICADA

KEIO UNIVERSITY

MATH. SCIENCES RESEARCH INSTITUTE

NEW MEXICO STATE UNIV.

OREGON STATE UNIV.

\author{
STANFORD UNIVERSITY \\ UNIV. OF BRITISH COLUMBIA \\ UNIV. OF CALIFORNIA, BERKELEY \\ UNIV. OF CALIFORNIA, DAVIS \\ UNIV. OF CALIFORNIA, LOS ANGELES \\ UNIV. OF CALIFORNIA, RIVERSIDE \\ UNIV. OF CALIFORNIA, SAN DIEGO \\ UNIV. OF CALIF., SANTA BARBARA
}

\author{
Daryl Cooper \\ Department of Mathematics \\ University of California \\ Santa Barbara, CA 93106-3080 \\ cooper@math.ucsb.edu \\ Jiang-Hua Lu \\ Department of Mathematics \\ The University of Hong Kong \\ Pokfulam Rd., Hong Kong \\ jhlu@maths.hku.hk
}

\section{Jie Qing}

Department of Mathematics

University of California

Santa Cruz, CA 95064

qing@cats.ucsc.edu

These supporting institutions contribute to the cost of publication of this Journal, but they are not owners or publishers and have no responsibility for its contents or policies.

See inside back cover or pacificmath.org for submission instructions.

The subscription price for 2012 is US \$420/year for the electronic version, and \$485/year for print and electronic.

Subscriptions, requests for back issues from the last three years and changes of subscribers address should be sent to Pacific Journal of Mathematics, P.O. Box 4163, Berkeley, CA 94704-0163, U.S.A. Prior back issues are obtainable from Periodicals Service Company, 11 Main Street, Germantown, NY 12526-5635. The Pacific Journal of Mathematics is indexed by Mathematical Reviews, Zentralblatt MATH, PASCAL CNRS Index, Referativnyi Zhurnal, Current Mathematical Publications and the Science Citation Index.

The Pacific Journal of Mathematics (ISSN 0030-8730) at the University of California, c/o Department of Mathematics, 969 Evans Hall, Berkeley, CA 94720-3840, is published monthly except July and August. Periodical rate postage paid at Berkeley, CA 94704, and additional mailing offices. POSTMASTER: send address changes to Pacific Journal of Mathematics, P.O. Box 4163, Berkeley, CA 94704-0163.

PJM peer review and production are managed by EditFlow ${ }^{\circledR}$ from Mathematical Sciences Publishers.

\section{PUBLISHED BY}

\section{mathematical sciences publishers}

http://msp.org/

A NON-PROFIT CORPORATION

Typeset in LATEX 


\section{PACIFIC JOURNAL OF MATHEMATICS}

Volume $260 \quad$ No. $2 \quad$ December 2012

\section{Special issue \\ devoted to the memory of Jonathan Rogawski}

In memoriam: Jonathan Rogawski

257

DON BLASIUS, DINAKAR RAMAKRISHNAN and V. S. VARADARAJAN

$p$-adic Rankin $L$-series and rational points on CM elliptic curves

261

Massimo Bertolini, Henri DARMON and KARTIK PRASANNA

The syntomic regulator for $K_{4}$ of curves

AMNON BESSER and ROB DE JEU

Unique functionals and representations of Hecke algebras

381

BENJAMIN BRUBAKER, DANIEL BUMP and SOLOMON FRIEDBERG

A relative trace formula for PGL(2) in the local setting

395

BROOKE FEIGON

On the degrees of matrix coefficients of intertwining operators

433

TOBIAS FINIS, EREZ LAPID and WERNER MÜLlER

Comparison of compact induction with parabolic induction

457

Guy HENNIART and MARIE-FranCE Vigneras

The functional equation and beyond endoscopy

497

P. EDWARD HERMAN

A correction to Conducteur des Représentations du groupe linéaire

HERVÉ JACQUET

Modular $L$-values of cubic level

ANDREW KNIGHTLY and CHARLES LI

On occult period maps

STEPHEN KUDLA and MiCHAEL RAPOPORT

A prologue to "Functoriality and reciprocity", part I

ROBERT LANGLANDS

Truncation of Eisenstein series

EREZ LAPID and KeITH OUELLETTE

Some comments on Weyl's complete reducibility theorem

JONATHAN ROGAWSKI and V. S. VARADARAJAN

On equality of arithmetic and analytic factors through local Langlands correspondence

FREYDOON SHAHIDI 